\title{
Negociaciones de identidad en la radio indígena y étnica colombiana: tres casos de estudio*
}

\author{
Enrique Uribe-Jongbloed ${ }^{\star *}$ \\ María Fernanda Peña Sarmiento***
}

Recibido: mayo 20 de 2014 - Aceptado: septiembre 13 de 2014

\section{Resumen}

El tema de la radio indígena y étnica, como parte de la falta de investigación sobre la radio en Colombia, ha sido poco desarrollado (Cuesta Moreno, 2012a, 2012b). Este artículo presenta tres casos de estudio: Radio Nasa de la comunidad Nasa, Jujunula Makuira de la comunidad Wayuu y el programa Al a with a wan de la comunidad Raizal del archipiélago de San Andrés, y explora cuáles son las perspectivas de identidad que encuentran espacios de negociación en la producción radiofónica, en particular en la relación de su identidad colectiva y cómo esta afecta o determina su uso lingüístico. La investigación surge de un proceso de tres pasos en donde los encargados de producción, las comunidades de práctica (Wenger, 1998), reflexionan sobre su labor y conceptualizan cómo se ve reflejada su identidad a partir de la construcción temática de su parrilla de programación. Los resultados evidencian que distintos niveles de filiación a varias de las facetas de identificación llevan a relacionar de diversas maneras la labor comunicativa del medio radial, y que estas identificaciones presentes en las comunidades de práctica tienen un efecto real sobre la producción radial lingüística de cada una de ellas.

Palabras clave: identidad, idiomas minoritarios, radios indígenas, cultura, etnia, participación, negociación e inclusión.

* Este artículo deriva parcialmente de la investigación COM-56-2011 Negotiation of identities in Minority Language Media production: Applying the perspective of Communities of Practice inscrita en la Universidad de La Sabana.

** Profesor Asistente, Facultad de Comunicación, Universidad de La Sabana. Campus Puente del Común, Chía, 250001, Colombia. Correo electrónico: Enrique.uribe@unisabana.edu.co

*** Profesora Asistente de la Facultad de Ciencias de la Comunicación de la Universidad Minuto de Dios. Correo electrónico: mfpena@uniminuto.edu 


\title{
Identity Negotiations on the Native and Ethnic Colombian Radio: Three Study Cases
}

\begin{abstract}
The topic of native and ethnic radio, as part of the lack of research on radio in Colombia, has been poorly developed (Cuesta Moreno, 2012a, 2012b). This article shows three study cases: Radio Nasa of Nasa Community, Jujunula Makuira of Wayuu Community, and Al A With A Wan program of native community from San Andrés island; it also explores the identity perspectives that find negotiation spaces in radio production, especially the relationship of their collective identity and the way it affects or determines its linguistic use. This research is based on a threestage process where producers (practice communities) (Wenger, 1998) make a reflection on their function and issue concepts on the way their identity is reflected from the construction of their programming. Results show that several levels of affiliation to several identification facets make it possible to relate, in several manners, the communication work of radio and that these identifications present in the practice communities have a real impact on the linguistic radio production of each community.
\end{abstract}

Key words: Identity; minority languages; native radio; culture; ethnic group; negotiation; inclusion. 


\section{Introducción}

El tema de los medios de comunicación minoritarios, sean estos étnicos, indígenas o lingüísticos, ha adquirido prominencia recientemente en las esferas académicas como consecuencia de un giro de interés por la identidad individual y colectiva, y por el desarrollo de tecnologías de comunicación de bajo costo, entre otras varias razones (Browne $\mathcal{E}$ UribeJongbloed, 2013; Browne, 2005; Castells, 2005; Castells-Talens, Ramos Rodríguez, \& Chan Concha, 2009; Husband, 1994; Riggins, 1992; Wilson \& Stewart, 2008). El surgimiento de la radio indígena en Colombia fue parte de un proceso de concertación entre comunidades indígenas y el Gobierno, continuando la exigencia que para tal fin se había consignado en la Constitución de 1991 (Rodríguez \& El Gazi, 2007; Unidad de Radio, 2002). Aunque la radio indígena surge a partir de un programa del Gobierno nacional a través del entonces Ministerio de Comunicaciones - hoy Ministerio de las Tecnologías de la Información y Comunicación- y del Ministerio de Cultura, las comunidades indígenas han podido usar estos medios como un espacio propio para presentar visiones alternativas a aquellas de los medios masivos privados y públicos, tal como lo presenta el caso de Radio Payu'mat de la comunidad Nasa en el Cauca (Murillo, 2008).

Si bien la radio indígena representa un campo de estudio interesante desde diversas perspectivas que incluyen la antropología, los estudios culturales, un abordaje de otras perspectivas epistemológicas sobre el concepto mismo de la comunicación, y los estudios sobre desarrollo, al igual que la radio colombiana en general, ha sido un campo que no ha sido explorado a fondo (Cuesta Moreno, 2012a, 2012b). Fuera de eso, aunque en Europa (ver Cormack, 1998, 2004, 2007, 2012) y en Australia (ver Forde, Foxwell, \& Meadows, 2009; Meadows \& Molnar, 2002; Meadows, 1992, 2009) se ha debatido en esferas académicas la relevancia de los medios de comunicación para ayudar al mantenimiento de las lenguas minoritarias, en Colombia este no ha sido el caso. Aquí se ha estudiado principalmente desde la perspectiva antropológica o los estudios étnicos, separando así los casos de minorías lingüísticas a partir de su filiación indígena o étnica.

Este artículo busca participar en la construcción de debate académico en torno al desarrollo de la radio indígena y étnica colombiana, planteando como caso de estudio dos emisoras pertenecientes a los grupos indígenas más numerosos en términos de población, los Wayuu y los Nasa, y un programa radial de la comunidad Raizal del archipiélago de San Andrés. Lo que tienen estos grupos en común es que son minorías étnicas o indígenas que son también minorías lingüísticas, cuyos idiomas se encuentran ya en algún nivel de riesgo de extinción'

Los tres casos planteados buscan presentar las diferentes características existentes en la producción mediática de las minorías, ya que estas tienen un doble rol de periodistas/comunicadores y activistas, las cuales no siempre son claramente compatibles (Husband, 2005). No obstante el rol de los medios en la preservación lingüística no sea claro (Cormack, 2007, 2012), es evidente que el uso de medios de comunicación por parte

1 Para conocer el nivel específico de peligro de cada lengua, revisar Lewis, Simons, \& Fenning (2013) 
de grupos minoritarios es una forma para reivindicar y contrastar las visiones hegemónicas que puedan existir sobre sus grupos sociales (Evans, 2002).

De acuerdo con un diagnóstico de la radio indígena en Colombia, de las 29 emisoras denominadas como indígenas, 21 realizan alguna parte de su transmisión en lengua indígena, 3 no transmiten en su lengua, aunque se usa por ese grupo indígena, y 5 transmiten solo en Español (ONIC, 2009). El diagnóstico no define el tipo de programación, o la cantidad o estilo de espacios en la parrilla de programación que son transmitidos en la lengua indígena, y por lo tanto, no da una visión completa de la presencia del idioma en su producción radial.

Para comprender los lineamientos bajo los cuales el equipo de producción indígena, y de igual manera en el caso de la producción étnica en San Andrés, define la inclusión o exclusión del idioma en su producción radial, es necesario comprender qué asociaciones establecen los productores radiales entre su identidad y su idioma.

\section{Negociaciones de identidad}

La producción radial indígena y étnica se inscribe entonces como un doble rol de ayuda a la preservación de la cultura y como una herramienta para la generación de esferas pública más pequeñas2 (Cunningham, 2001; Gitlin, 1998) de debate propio y con la cultura mayoritaria. Esta disyuntiva nos lleva a preguntarnos sobre cómo se desarrolla su actividad lingüística, y cómo se privilegia, remueve o presenta la lengua en el espacio radial. Para ello, habría que preguntarse sobre el rol que desempeña la lengua para aquellos que participan en la producción de contenidos radiales.

Pavlenko y Blackledge (2003) hablan de las negociaciones de identidad como aquellos procesos de asignación, apropiación y redefinición de la identidad que transcurren en la relación intercultural, en especial en casos de identidades minoritarias en relaciones con las mayoritarias. Ellos plantean tres tipos de identidad: impuesta, cuando la identidad ha sido definida por la mayoría sobre la minoría y surge de un interés de control o dominación (asignación racial colonial, por ejemplo); asumida, cuando una identidad otorgada por la mayoría es usada por la minoría para agruparse como iguales en su diferencia, diría GarcíaCanclini (2004) y buscar oposición a las visiones hegemónicas que les son impuestas (el caso de la re significación del concepto niche en Colombia, o el movimiento indígena en Latinoamérica); y negociada, en aquellos casos que la minoría construye y redefine su identidad, evita plantearla en oposición a la mayoritaria y la determina de manera propia.

Esta categorización es útil para comprender cómo se ubican los productores radiales indígenas y étnicos ante su propuesta lingüística y cultural, pues al saber qué tipo de identidad presentan y cómo la ven plasmada en su parrilla radial, podremos tener mayor claridad sobre cómo conciben su uso mediático como parte de su cosmovisión.

2 Cunningham (2001) y Gitlin (1998) usan el término en inglés sphericules para definir estas pequeñas esferas públicas dentro de, o en contacto con, la esfera pública nacional. 


\section{Metodología}

El primer paso fue determinar los grupos indígenas o étnicos para incluir en el estudio. Basado en la cantidad de usuarios del idioma minoritario y tratando de representar al menos un grupo indígena y uno étnico no indígena, se seleccionaron Jujunula Makuira (Comunidad Wayuu, ubicada en Nazareth, alta Guajira), Radio Nasa de Tierradentro (Comunidad Nasa, Cauca) y La voz de las Islas (Comunidad Raizal, Archipiélago de San Andrés). Cada una de esas comunidades tenía, a 2011 que fue la fecha de la realización del trabajo de campo, acceso a una emisora que, al menos potencialmente, podría transmitir en sus lenguas minoritarias respectivas (Wayunaiki, Nasa Yuwe y Creole).

Para comprender la relación entre la producción, la identidad y el idioma, se llevó a cabo un proceso de triangulación metodológica que involucró tres instrumentos. El primero fue una entrevista con el doble (ITTD, por su sigla en inglés), según la propuesta metodológica definida por Nicolini (2009). Este proceso se lleva a cabo pidiendo a los productores radiales que describan un día completo de su trabajo, como si fuesen a contratar un doble que los remplazase por ese día en todas sus funciones. La idea de esta entrevista es obtener una noción sobre aquello que consideran cotidiano, básico y no problemático en su labor diaria, pero clave y necesario para que su labor se cumpla satisfactoriamente.

Después de esta entrevista se realizó una observación al equipo de producción durante un día de trabajo. En dicha observación los investigadores cotejaron la información presentada por ellos en la ITTD con aquello que veían en su desempeño real. De este modo se evidenciaban contradicciones, omisiones y aspectos que quizá no habían sido valorados por los productores como relevantes originalmente. Estos aspectos se mencionaban a los informantes in situ de modo que esto generase una reflexión inmediata que nutriría la entrevista posterior.

Finalmente se desarrolló una segunda entrevista. Esta fue una entrevista semiestructurada que traía a colación las similitudes y diferencias entre el ITTD y la observación realizada, con el interés de comprender cómo determinaban el espacio lingüístico en su parrilla de producción.

La entrevista se desarrolló de manera individual o grupal, dependiendo de las posibilidades de tiempo de cada uno de los grupos de producción.

Cabe mencionar que el caso del equipo de producción raizal en San Andrés es considerablemente diferente. La Voz de las Islas, emisora que al momento de la impresión de este artículo ya no existe en el dial, era en su momento la única emisora legal transmitiendo en lengua creole en San Andrés. El equipo entrevistado fue exclusivamente de aquellos productores que trabajaban en los programas radiales en Creole que producía dicha emisora.

\section{Resultados}

Los resultados de los tres casos de estudio son presentados por separado, pues sus cualidades y consideraciones son diferentes. Al final se traerán a colación los elementos de discusión de las tres partes. 


\section{Jujunula Makuira \\ Entrevista con el doble (ITTD)}

Tal como se podía esperar de esta metodología, la entrevista con el doble proveyó una idea general de las prácticas rutinarias de los productores de esta emisora. Los pasos de procedimientos para el funcionamiento de la estación radial fueron explicados por los participantes y son presentados con lujo de detalle, consignado aquí en la siguiente lista:

1. Verificar el fluido de la planta eléctrica del pueblo. En caso que no se haya activado la planta del pueblo, encender la planta eléctrica de diésel de la emisora.

2. Prender y activar todos los equipos (transmisor, sistema de aire acondicionado, computadores, moduladores estéreo).

3. Cinco minutos antes de comenzar la programación, a las 6 p. m., poner los audios de identificación de la emisora.

4. A las 6 p. m., transmitir el himno nacional de la República de Colombia.

5. Emitir el cabezote del espacio "Momento Cultural".

6. Transmitir una grabación de un instrumento musical Wayuu.

7. Invitar a la audiencia a sintonizar.

8. Entre 6 p. m. y 7 p. m. transmitir un cuento pregrabado, seguido de un comentario sobre el mismo, y un Jayechi (historia cantada) seguido de su comentario.

9. De 7 p. m. a 8 p. m. hay un espacio para la participación de la audiencia, para un invitado o para mensajes locales.

10. De 8 p. m. a 9 p. m., incluso hasta las 9:30 p. m, se presenta música contemporánea.

11. Después de las 9 p. m., cuando se termina la transmisión, apagar los equipos y la planta eléctrica, si esta se hallaba prendida.

Esta es la rutina típica descrita en la entrevista. Sin embargo, hay algunos elementos interesantes que vale la pena mencionar. Primero que todo está el asunto del himno nacional (ver punto 4 en la tabla), el cual se presenta en dos idiomas. Uno de los participantes asegura esto diciendo que "a las 6 en punto tengo que colocar el himno nacional y el himno departamental, [lo cual] es en Wayuunaiki y en lengua castellana [SIC]" (ITTD Jujunula Makuira, Informante 2).

Más adelante, ambos se enfocan en la conexión de un instrumento musical que ellos consideran como parte de su cultura. Ellos mencionan estos instrumentos (Kashaa, Torompa o Massi) y los describen como instrumentos Wayuu. Esto recalca la relación de la programación que usa estos instrumentos como cortinilla, a la que ellos denominan como su Momento Cultural (ver los puntos 6 a 8 arriba).

El primer saludo después del instrumento musical es específicamente en wayuunaiki: 
Hablas en wayuunaiki, saludando, dando la bienvenida. Te expresas a la comunidad, mencionas la emisora, saludas de paso a las comunidades en wayuunaiki (ITTD Jujunula Makuira, Informante 1).

La relevancia de entregar un contexto y una interpretación al cuento y al Jayechi es enfatizado por ambos participantes, uno de ellos mencionando que "siempre te tienes que mantener expresando en Wayuunaiki, en este espacio" (ITTD Jujunula Makuira, Informante 1), y aclarando que esto se hace hasta las 7 p. m.

Durante la emisión después de las 7 p. m. las aclaraciones principales que hacen versan sobre los mensajes de y para la comunidad, o los mensajes de interés público:

(...) te los hacen llegar en castellano. El mensaje en castellano, tú tienes que traducirlo a wayuunaiki para que algunas personas lo entiendan también (ITTD Jujunula Makuira, Informante 1).

$\mathrm{y}$

(...) Más que todo a los avisos sociales tenemos que pasarlos en lengua castellana y luego explicarlos en wayuunaiki para que los oyentes los entiendan más (ITTD Jujunula Makuira, Informante 2).

Después de las 8 p. m., uno de los productores comenta que "si no están los otros compañeros, tú puedes continuar con la variedad de música en castellano, y eso, vallenato, merengue, salsa, tú la vas variando" (ITTD Jujunula Makuira, Informante 1). Después del programa musical, la radio cierra por el día.

\section{Entrevista semi-estructurada tras la observación}

El primer aspecto que es consistente es que la transmisión en wayuunaiki está casi completamente concentrada en la hora de programación de 6 p. m. a 7 p. m., no obstante el compromiso con su manutención y con su identidad lingüística. Durante la entrevista, en varias ocasiones la división de la programación en la parrilla fue mencionada como dependiente del idioma, separando las afiliaciones de los programas en contenido e idioma simultáneamente:

Informante 1: Prácticamente en las grabaciones que hacemos, que nosotros tenemos ahí, que diga 'Sintonízate conmigo a través del dial en el 90.2', ahí también la tenemos en wayuunaiki. Pero esa identificación en wayuunaiki nosotros la pasamos en el espacio que nosotros estamos emitiendo la programación en wayuunaiki. Sería una forma de trabajar de nosotros: de que nosotros emitimos la identificación en castellano, y la de wayuunaiki nosotros la emitimos en el espacio de wayuunaiki (ES Jujunula Makuira).

Esta división de la transmisión lingüística crea marcadores de identidad cerrados y delimitados. El espacio del wayuunaiki se hace idéntico a aquel de la programación de la cultura Wayuu que ellos denominan Momento Cultural. Allí presentan los instrumentos musicales, sus cuentos y Jayechis con los que identifican lo Wayuu. De este modo queda claro cómo reconstruyen esa visión de identidad de qué es Wayuu a través de los instrumentos musicales: 
Informante 1: Mediante eso, nosotros emitimos lo que es los instrumentos musicales que nos identifica también, más que todo el kashaa, el maasi...

Informante 2: Torompa

Informante 1: El torompa es un poquito, como digamos occidental, porque eso viene hecho de hierro. El guaguay. Hay dos clases de guaguay, [uno] que uno lo puede hacer con la mano, y otra tiene que ser de una planta; el fruto de una planta [al] que uno le hace unos huequitos (ES Jujunula Makuira 1).

Al definir el Torompa como menos representativo de la cultura Wayuu por su material y las connotaciones del mismo, la identificación Wayuu se ve como fija en el tiempo previo al contacto con la cultura occidental. De este modo se evidencia la identificación de los productores como una visión esencialista de la separación étnica.

Un caso similar es el de los cuentos de los ancianos en la sección en wayuunaiki de la programación. Los participantes se adhieren a una identificación conectada con la relevancia de las historias de los ancianos, basados en los consejos y palabras de sabiduría que estos proveen:

Informante 1: (...) El anciano, él te echa, él te cuenta un cuento, con unos términos... o sea, hay unos términos, que son como un consejo de él. [Donde] él dice que no es bueno robar, que eso es malo robar, y que esto, y entonces, mientras está echando el cuento (ES Jujunula Makuira 1).

Pero como la mayoría de los cuentos son pregrabados, ellos escogen apartes para resumir o extraer el consejo, con un énfasis especial, en algunos de ellos, para niños:

Informante 1: (...) O sea que, ellos cuentan que una vez el conejo era una persona que esto, y hacía esto, y por mentiroso quedó así. Entonces, en esa parte es que uno escoge lo que es el consejo, o sea, hace como un pequeño resumen y uno dice que no es bueno decir la mentira. Si, esto es para llamarle la atención a los niños, más que todo a los niños que están estudiando hoy en día (ES Jujunula Makuira 1).

Ellos se apropian de este estilo narrativo como una identificación de la ética y el comportamiento apropiado para el grupo. Es muy diciente, por lo tanto, que son los ancianos mismos quienes valoran más este tipo de historias, según lo presentan los informantes:

Informante 1: (...) Algunos ancianos si han querido, han querido escuchar los cuentos. Ellos, a través de algún nieto ellos envían, ellos dicen 'no, que de parte de mi abuelo, para que pases el cuento del conejo' -digamos así un ejemplo-. Entonces nosotros lo pasamos (LI Jujunula Makuira 1, p. 7).

No obstante la autoridad de los ancianos, algunas historias son cuestionadas por la audiencia con respecto a su contenido. En algunos casos evidencian su desacuerdo con el tipo de historia que se cuenta, poniendo en entredicho la identificación de la sabiduría de los ancianos, y evidenciando el problema de la división lingüística de la programación con respecto a los horarios de transmisión:

Informante 1: (...) Una vez nos llamaron telefónicamente, una muchacha joven. 
Ella nos llamó por teléfono porque (...) en el cuento, es que él emitía que su comadre, o sea, estaba muy buena, pues, en el cuento, o sea, pero en wayuunaiki (...) Entonces él aprovechó de que su comadre estaba durmiendo y se acostó con ella. O sea, ahí hay unos términos que son así como...

\section{Informante 2: Términos vulgares}

Informante 1: Si, o sea, como términos vulgares. Entonces la muchacha llamó ese día y dijo 'oye, hay niños que están escuchando ese cuento, por lo tanto no sería, yo creo que no sería muy bueno emitir ese cuento porque tiene unos términos así' (...) Si, y hay otro cuento, hay otro cuento también que es en wayuunaiki (...) Por ese lado también nos llamaron la atención. '¿Por qué emiten cuentos así que estén en términos vulgares?' O sea, la comunidad también nos ha... nos ha llamado la atención por ese lado (SE Jujunula Makuira 1).

El interés en promover la cultura y el idioma Wayuu, según lo presentaron los participantes, no se evidencia tan claramente en la programación de la emisora. De hecho, hay referencias constantes a la oposición popular a la presencia de formas tradicionales de producción cultural. Como se señalaba arriba, en el caso de cuentos algo vulgares, hay reacciones similares mencionadas por los productores con respecto a la duración del Jayechi (cuento cantado tradicional) o la duración de los discursos de los ancianos que transmiten en vivo. En contraste con el interés de los ancianos, los productores afirman que los jóvenes se interesan más por la sección de música contemporánea durante la sección en castellano de la programación. Esto hace fácil el entender por qué "los estudiantes siempre prefieren la programación en castellano" (SE Jujunula Makuira 1).

Más allá de la contraposición a la autoridad de los ancianos, la narrativa personal de los productores de la emisora explica de mejor manera la aproximación de la emisora:

Informante 1: Muchos jóvenes, muchos niños han accedido a Venezuela que es el país que está más cerca. Y tienen una entrada como de digamos libre. El wayuu apenas tiene 10 años, 14 años ya sale para Maracaibo. En Maracaibo conoce lo que es el trabajar. Hasta los niños trabajan. Trabajan allá y ellos se van amañando y se van acostumbrando a la cultura de allá. Digamos así. Y así va perdiendo lo que es su idioma, lo que es su cultura (...) yo prácticamente no nací aquí en la alta Guajira, yo nací es en Venezuela. A los 8 años llegué aquí, no sabía hablar el wayuunaiki (...) Mi abuelita, que en paz descanse, ella fue la que me enseñó a aprender lo que es el respeto, lo que es la cultura, a hacer lo que tenía que hacer, lo que es en el pastoreo, y todas esas cosas... o sea, lo anterior (SE Jujunula Makuira 1).

La división lingüística-cultural en identificaciones separadas es parte de una perspectiva personal que se ratifica en el proceso de producción. Cuando se les preguntó por la falta de producción en wayuunaiki, la respuesta primordial de los participantes fue la falta de recursos para viajar y recolectar más información de los ancianos que viven lejos de la emisora. Este interés se hace evidente en dos apartes de la entrevista:

Informante 1: (...) Como le estaba diciendo yo, quisiera hacer los programas así que todo sea en wayuunaiki. Pero para eso yo tengo que salir a investigar (...) Si un anciano muy conocido allá en Siapana, trae un cuento, nos cuenta un cuento, (...) comparando la 
comunidad de aquí, la comunidad de Siapana con la de acá, comparando los cuentos que cuentan ambos viejos. Yo creo que veríamos una diferencia ahí, habría una diferencia ahí entre esos dos, no muy distintos, pero sí habría una diferencia (SE Jujunula Makuira 1).

(...) Sí, como te estaba diciendo, yo quisiera que toda la programación fuera en wayuunaiki, pero uno tendría que salir a trabajar, a investigar para uno poder tener diferentes informaciones, y también tener diferentes informaciones de otras personas, que no sea de una sola persona (SE Jujunula Makuira 1).

Fuera del escaso presupuesto, también hay algunos problemas como lo son la falta de conocimiento sobre la forma estandarizada del idioma, bien sea hablado o escrito, que se convierten como argumento para el uso del castellano en vez del wayuunaiki. Además, el programa entre las 7 p. m. y 8 p. m. incluía una sección de Salud y Bienestar preparada por el personal del centro médico local,

Informante 1: Pero lo hacían todo en castellano. Entonces la idea de nosotros era de traducirlo también de que ellos lo dijeran en wayuunaiki porque ellos le están mandando el mensaje a una comunidad wayuu no a una comunidad alijuna3 sino a una comunidad wayuu. Y ahí siempre nos ponen peros con respecto a eso: 'No, que el wayuunaiki es muy difícil pronunciarlo', 'no, que me equivoco mucho en wayuunaiki. Es mejor así, en castellano' (SE Jujunula Makuira 1).

Esto no es sorprendente, pues ambos productores mencionan que sus competencias en wayuunaiki escrito son muy bajas. Uno de ellos asegura que escribe primordialmente en castellano, aunque sabe pronunciar el wayuunaiki, mientras que el otro productor asegura que escribe en castellano porque se confunde al hacerlo en wayuunaiki.

Como se presentó arriba, ellos muestran marcadores de identidad interna y externa del grupo que los distingue a ellos como wayuu, y a los otros como alijuna. No obstante esa diferencia, ellos también quieren llegar a una audiencia alijuna a través de su producción radial, según lo presentan en su entrevista.

\section{Radio Nasa}

La entrevista con el doble tuvo un giro interesante en el caso de Radio Nasa. Aunque ambas descripciones fueron considerablemente similares en la definición de las rutinas diarias, una incluyo más referencias a un contexto más allá del orden cronológico. Para la descripción de la labor diaria ellos usaron el horario de programación y establecieron las tareas importantes en relación con ella, presentando como relevantes los siguientes asuntos:

1. Llegada a la estación a las 5 a. m. para prender los equipos.

2. Revisar Internet y otras fuentes de información para nutrir la información de sus programas.

3. Programas radiales en vivo se llevan a cabo según la parrilla de programación.

3 La palabra Alijuna se refiere a aquellos que no son miembros de la etnia Wayuu, ni a ninguna de aquellas con las que los Wayuu han tenido contacto previo a la llegada de los occidentales. 
4. Compilación y edición de los elementos de los programas, las notas y anuncios recibidos por telefonía celular, mensajes de texto o visita personal de las personas interesadas.

5. Programación de una variedad de géneros: informativos, educativos, de música y entretenimiento.

6. Constante revisión y aprobación de los elementos a ser transmitidos.

El asunto del idioma no fue mencionado por los productores, y parece ser visto como no problemático. Mayor prominencia se le da a asuntos de organización, movilización y representación política. Ellos mencionan una variedad de programas como "un programa que va hasta las seis de la mañana que se llama Pueblos en Marcha que es un contenido político organizativo" (ITTD Radio Nasa, Informante 1) y "una especie de radio revista, va acompañado de música, humor y la noticia de actualidad, y también lo que está haciendo como, bueno, lo que están haciendo las organizaciones a nivel de aquí del municipio, a nivel departamental, nacional" (ITTD Radio Nasa, Informante 2).

También mencionan grupos de interés específicos que son fundamentales para ellos: autoridades tradicionales, cabildos, la alcaldía, la gobernación y también buscan tratar temas de interés para los " tres sectores, la población indígena, la población campesina y la población afro-colombiana. Entonces, en ese momento hablamos de los tres, de las tres, de los tres sectores, cual es el avance por el proceso político-organizativo de la comunidad Afrodescendiente, el mismo tema con las comunidades indígenas, con las comunidades Nasas" (ITTD Radio Nasa, Informante 1), y con las organizaciones campesinas. También abarcan temas sobre " prevención en la parte de salud" " (ITTD Radio Nasa, Informante 2). Además, enfatizan su colaboración con redes de comunicación indígenas de la región, incluyendo la producción de grabaciones de noticias de 10 a 20 minutos para ser distribuidas por sus estaciones afiliadas.

\section{Entrevista semi-estructurada y observación}

El aspecto del idioma no fue mencionado en ninguna de las entrevistas con el doble. La observación permitió establecer que solo había dos tipos de programas hablados en vivo que se transmitían primordialmente en Nasa Yuwe. Estos incluían pequeños elementos grabados en castellano que eran resumidos en Nasa Yuwe, o entrevistas largas en Nasa Yuwe con breves resúmenes en castellano. No obstante, la programación en castellano, en su mayoría en las secciones de entretenimiento y música, no incluía resúmenes en Nasa Yuwe. Esto evidencia que Radio Nasa rara vez alcanza el balance mencionado en la entrevista semi-estructurada, donde afirmaban que "la emisora, inicialmente cuando empezó, el porcentaje era casi que el cincuenta y cincuenta, cincuenta (por ciento] en Nasayuwe, y 50 [por cientol español" (SE Radio Nasa 1).

Al hablar sobre uno de los programas de radio presentados por uno de los productores y observado por los investigadores, surgió una pregunta sobre el balance de los idiomas, 
Nasa 2: Por lo general nosotros manejamos cincuenta-cincuenta. Cincuentacincuenta porque a esta hora ya la gente, los campesinos, o sea, toda la gente que labora en el campo ya viene, ya regresa a casa y sobretodo - como te había dicho-, este proyecto es para, para... de los cabildos. Es un proyecto de los cabildos. Igual, el carácter de la emisora es público, entonces manejamos para todos. Ahí está para los Nasas y para los que hablan español, entonces precisamente manejamos los... yo manejo los dos idiomas, para que el que habla español, si solo hablo Nasa Yuwe, pues no me va a entender, entonces si lo hago en dos idiomas, las cosas van a quedar claras (SE Radio Nasa 2).

El productor se refiere a los Nasa como una categoría diferente a aquellos que hablan castellano, aunque después asegura hablar ambos idiomas. No obstante, otro integrante menciona que "en la emisora, de planta trabajamos 3 personas, dos personas hablan Nasayuwe, yo sí hablo el español" (SE Radio Nasa 1). Debido a la diferencia en las habilidades lingüísticas de los productores, los programas son bilingües castellano-nasa yuwe o exclusivamente en castellano.

Con respecto a la música, hay un énfasis en la música regional, considerando la región de Tierradentro como la fuente principal de música presente en la emisora:

Nasa 2: En el proyecto de comunicación indígena como tal nos identificamos por la música puesto de que en Tierradentro hay mucho talento. Hay muchos músicos, hay hartísimos músicos. Nosotros tenemos un programa especialmente para presentar música, música de la región, o artistas de la región. Que es música de la región también porque habla de la vida de los pueblos indígenas, habla del tema de la resistencia, habla de la minga, habla de la evolución de los jóvenes, habla de la actualidad, de la modernidad pero desde la ... desde nuestros artistas (SE Radio Nasa 2).

También hay un pequeño porcentaje de música diferente, la cual se define por exclusión como música que no es Nasa, y otros tipos de música, en especial canciones de protesta y de movimientos sociales.

Nasa 1: Después de las seis va, va mucha información pero con mucha música, ya más enfocada a lo occidental, o más enfocada también a otras culturas, como el vallenato, la salsa; en sí, muy variadita ya la programación. Al mediodía también tenemos un programa ya netamente que es, pues enfocado a la cultura, conocer no solamente la cultura de aquí, sino de otros pueblos: lo andino, lo social, música social que nos enseña a convivir y a reflexionar y a vivir más (SE Radio Nasa 1).

La música se separa, entonces, en tres categorías: música regional, música social/ andina, y música occidental/otra. La música regional es asociada directamente con Radio Nasa en términos de tema, contenido y localidad; la música social/andina es similar en cuanto a contenido y las enseñanzas que presentan mientras que la música occidental/ otra es considerada simplemente como entretenimiento.

Fuera de eso, la identificación más relevante, como lo evidenciaba la entrevista con el otro es la política, en especial el tema de resistencia territorial y cultural que promueve la emisora. Hay menciones claras sobre la relevancia de los derechos humanos y territoriales como parte de los contenidos de los programas: 
Nasa 2: (...) manejamos mucho el tema de Derechos Humanos. (...) Hay una problemática sobre el tema de minas, sobre el tema del control territorial, bastante complicado hacia el occidente del departamento del Cauca, más exactamente donde está el resto de compañeros Nasas, hermanos Nasas, para el municipio de Suárez. (...) Entonces, en 15 resguardos son, es mucha la tierra que hay solicitada por la Anglo Chanti, entonces, precisamente por eso se maneja mucho el tema de control territorial, y el tema de derechos humanos (SE Radio Nasa 2).

Esta identificación política también incluye mayor intercambio de información sobre las autoridades indígenas y otros movimientos y organizaciones indígenas.

Nasa 1: Cuando recién iniciamos no sabíamos ni que era una organización, ni que era el CRIC ni qué era la ONIC. Ahora, ya se tiene conocimiento, y quiénes nos representan. Qué función debe hacer un gobernador. Qué función tiene la asamblea para elegir un gobernador. Se ha dado conciencia para las personas para la comunidad, para aprender a querer y saber elegir a los que nos van a representar (SE Radio Nasa 1).

De este modo, mostrando la idea de que el interés principal de la estación radial se identifica con el aspecto político-territorial de la cultura Nasa, y sobre cómo es este su elemento base:

Nasa 2: Nosotros siempre hemos dicho, la emisora para las comunidades indígenas de la región de Tierradentro ha sido una herramienta fundamental porque ha sido como del silencio a la palabra. Me refiero del silencio a la palabra porque anteriormente, antes de la radio se hacían trabajos importantísimos así como los que se hacen ahorita, reuniones, asambleas, propuestas, proyectos sobre el tema de control territorial, sobre el tema de minas, sobre el tema de aguas, de cómo manejar el tema de la salud propia, la educación propia, la implementación de la educación propia, el fortalecimiento del Nasa Yuwe, toda esa parte (...) entonces ha sido un aporte importante el tema de la concienciación, de que sí tenemos tierra, de que nosotros sí valemos, de que nosotros sí tenemos nuestros derechos, de que sí hay leyes que nos defienden. (SE Radio Nasa 2).

Además, esta identificación política es ampliada para llegar a otros participantes más allá de la comunidad indígena, y acercarlos como compañeros importantes en la movilización y resistencia a la asimilación cultural mayoritaria:

Nasa 1: El caso de las cuatro de la tarde hay un programa que se llama "Voces de identidad y autonomía". Como el mismo nombre lo dice, es para que las diferentes organizaciones se expresen a través del medio y enseñen, no solamente al indio, o al negro, o al campesino, de su organización solamente ellos, sino conocerlo en general, y tratar de unir esas culturas, no dividirnos, como siempre ha pasado (SE Radio Nasa 1).

Finalmente, la falta de presencia del nasa yuwe en la programación se presenta como un problema de recursos y competencias disponibles. Reportan una falta de financiación para emplear más productores; en esencia, abrir una convocatoria para una mujer. Uno de los participantes afirma:

Nasa 2: Por ese lado, en estos días se nos ha dificultado en buscar la persona que, en conseguir a la persona que necesitamos y prácticamente en este momento necesitamos es una mujer que hable Nasa Yuwe, que escriba Nasa Yuwe, y que lo entienda y que 
lo lea. Para que se pueda trabajar, como ya le dije, como el Nasa Yuwe ya lo manejamos cincuenta-cincuenta entonces necesitamos un equipo que sea fuerte sobre el tema de Nasa Yuwe. (LI Radio Nasa 2, p. 4).

Esto evidencia el interés en sobreponerse al sesgo masculino de la producción, al mismo tiempo que se aumente el personal bilingüe de la estación.

\section{La voz de las Islas}

Las entrevistas con el doble realizadas a los productores de dos programas de radio de La voz de las Islas no provee una descripción clara en términos cronológicos. Sin embargo, resalta algunos de los procedimientos básicos que son llevados a cabo por sus miembros en la producción de los programas. Un resumen de su trabajo de producción está a continuación:

1. Revisar los diferentes medios (internet, estaciones de radio nacionales, y televisión) buscando noticias nacionales, regionales y locales.

2. Contacto con la comunidad, a través de intercambios personales, sobre otros temas noticiosos.

3. Participación en el programa (usualmente 10:30 a 11:30, lunes a viernes, o $12 \mathrm{pm}$ a $2 \mathrm{pm}$ los sábados).

4. Recibir llamadas telefónicas de las personas que desean participar en el programa.

5. Si no pueden estar presentes durante el programa, participar por vía telefónica.

Un elemento principal es la decisión de ambos participantes de usar Creole como el idioma de su entrevista. Aunque uno de ellos incluyó también un resumen de la misma en castellano, ambos comenzaron con comentarios sobre el creole y el rol que desempeña en la estación radial. Esta introducción a su descripción de actividades incluye una demostración clara de su interés lingüístico. Ambos participantes presentan su caso en sus propias palabras:

Raizal 1: First of all, our language in this island is our Creole, our second language is English, then now the third language may be the Spanish language also, that the Colombian government come and impose on us native Caribbean people, the afroCaribbean (...) (ITTD La voz de las islas, 1).

Raizal 1: Primero que todo, nuestro idioma en esta isla es nuestro creole, nuestro segundo idioma es el inglés y nuestro tercer idioma puede ser el español también, que el gobierno de Colombia ha venido a imponernos a nosotros, la población Caribeña nativa, los afro-caribeños (...)

Raizal 2: Como venía diciéndoles en mi idioma creole en inglés, el programa 'Al a wii da wan' (...) es un programa, es un espacio para la comunidad raizal, la comunidad, ehh, que de pronto no tiene la capacidad de manifestar su inconformismo, de manifestar sus inquietudes, o de participar de una forma en los programes normales que hay en el idioma español, ya que el idioma español para nosotros es nuestro segundo idioma y 
no nuestro primer idioma, nuestro idioma es el creole, el inglés, ese es nuestro primer idioma, nosotros reconocemos el español como nuestro segundo idioma y por estatutos, llámese por ley, llámese por constitución (...) Bueno, el creole es nuestro idioma, y nuestro programa es netamente en creole, para las personas que hablan creole y tengan la oportunidad de interactuar con nosotros (ITTD La voz de las Islas, 2).

Ellos también expresan su indignación sobre la noticia acerca de la revocatoria de la licencia de uso del espectro electromagnético de la emisora (algo que tuvo efecto a finales de 2011) y ven este asunto como un problema para la audiencia que sirven. Presentan su caso de la siguiente manera:

Raizal 1: These people that want to close the oldest AM radio station in the whole archipelago, the radio station that make our native people in our archipelago can communicate themselves (...) they don't have no relative in our island, to come and want to close our radio station. They got closing our radio station, you are taking away our language, you are taking away totally the communication between our brothers and sisters also (...) (ITTD La voz de las islas 1. p. 1).

Raizal 1: Esta gente que quiere cerrar la más vieja estación en AM en el archipiélago entero, la estación de radio que permite a nuestra gente comunicarse (...) ellos no tienen parientes en nuestra isla, para venir aquí a cerrar nuestra estación. Si ellos cierran nuestra estación, estarían arrancándonos el idioma, estarían quitándonos la comunicación entre nuestros hermanos y hermanas (...)

Raizal 2: They are trying to close it, and close our voice, the voice of the creole people, the voice of the raizal people, the voice of the minority in this beautiful archipelago. They want to close it down through political's affairs and grudgeness (ITTD La voz de las islas 2, p. 1).

Raizal 2: Ellos están intentando cerrarla, y acallar nuestra voz, la voz de la gente creole, la voz de la gente raizal, la voz de la minoría en este hermoso archipiélago. Ellos quieren cerrarla a través de artimañas políticas y ambiciones personales .

La entrevista con el otro evidencia el conflicto político/lingüístico bajo el cual ellos consideran su trabajo de producción. Este conflicto político/lingüístico no descansa sobre temas lingüísticos, sino sobre otras políticas y reglamentaciones sobre las estaciones comerciales, pero el espacio para la identificación lingüística que otorga el espacio radial se hace el elemento fundamental de la estación para tratar de evitar su clausura.

\section{Entrevista semi-estructurada y observación}

Durante la entrevista semi-estructurada, después de participar en su programa de sábado 'Feelin' Eiry', ambos productores ofrecieron sus perspectivas sobre las negociaciones de identidad a lugar en la producción de sus programas radiales.

Ellos privilegian la posición del idioma creole en sus programas, enfatizando su pertinencia en distintos aspectos que lo hacen primordial en su identidad:

Raizal 2: Bueno, primero que todo, tenemos que basarnos en el idioma. Nosotros tenemos un idioma, que es nuestro primer idioma, que es creole. Y los temas que tra- 
tamos tienen que ver con lo que afecta directamente a la población raizal, entonces ya hay una identidad allí. (...) Entonces, refleja en lo cultural, primero que todo, porque, repito, es por el idioma. El idioma explícito se habla bien claro nuestro idioma creole, uno no trata de poner, uno no trata de poner tecnicismos ni nada, es un creole pleno (SE La voz de las Islas 1).

Pero también mencionan cómo su multilingüismo contribuye a mostrar características de la identidad raizal:

Raizal 1: Por eso siempre damos gracias a Dios, que nosotros aquí en esta región del caribe-San Andrés islas- aquí somos bilingües, trilingües, cuatrilingües, hasta cinco seis, siete, ocho. Cuando le queramos poner, y esa es la ventaja que tenemos nosotros, que podemos acercarnos a cualquier persona en su idioma cuando no conoce el nuestro. Pero si esa persona conoce mi idioma, y puede entenderlo y hablarlo, -olvídese-, voy con él en creole hasta que se termine el tema (SE La voz de las Islas 1).

Raizal 2: Sí, referente a la participación de personas de otro idioma, específicamente el español ¿no? Hay personas que viven en la isla que no hablan el creole, se les dificulta ¿no?, por el acento, hablar el creole. Pero entienden el creole. Llaman y cuando se toca un tema y es interesante para ellos, participan. Pero piden disculpas primero, que no pueden dirigirse en creole, pero van a hablar en español. (...) Entonces estas personas participan en su idioma, en su idioma español. Y son bienvenidos (SE La voz de las Islas 1).

Raizal 1: Muchas personas que ocupan cargos aquí en la isla, referente al turismo -digamos el gerente de alguna agencia de viajes, o algo por el estilo, no hablan el creole, no hablan el inglés. Y son gente que invitamos y hacemos una excepción, presentamos el programa, en español y hacemos un recuento en creole, para que la persona pueda desenvolverse, pueda llegar a que sea entendido por lo que va a anunciar al público presente (SE La voz de las Islas 1).

Este es el caso de la encapsulación del idioma creole, y la falta de espacios para su difusión; debido a que el interés del programa no es únicamente la manutención lingüística, sino asuntos de interés público, esta concesión se hace necesaria. De cualquier modo, al resumir en creole, el castellano no es visto como un idioma que puede ser necesariamente entendido por toda la audiencia. Así, la audiencia se ve como posiblemente monolingüe. En el caso opuesto, cuando el creole es el idioma en discusión, ellos hacen la misma concesión a favor del debate público. Pero contrario a la separación de espacios lingüís ticos de exclusividad mutua, ellos abogan por visibilidad y diferenciación clara, en vez de separación lingüística.

Raizal 1: Jugamos el papel del bilingüe, pero siempre tratamos de mantener siempre enfocado en nuestra cultura, en nuestro idioma, a la gente al día. ¿Por qué? El 99 $\%$ de los programas que se pasan en el canal regional son en español, igual dentro de la radio, ¿cierto?, y últimamente estamos tratando de que, 'bueno, pues si todos los otros programas son netamente en español, vamos a hacer algo netamente en creole', vamos a dirigirnos específicamente a nuestra gente. Pero nosotros de buen corazón, no podemos ser egoístas de esa manera, siempre omitimos algo, restamos algún porcentaje para que los de habla hispana también puedan por lo menos, 'bueno, yo ya sé por dónde van, por qué hablaban, o qué es lo que decían' (La voz de las Islas 1, p. 3). 
Su provisión de otros idiomas acepta y se nutre del capital multilingüe de ambos productores y de la audiencia, ampliando las posibilidades de comprensión lingüística, pero manteniendo la relevancia superior del creole. Al debatir sobre su identidad ellos respondieron:

Raizal 1: Que no debemos de ser egoístas en cuanto a cultura, porque ya San Andrés, ya no es una cultura netamente criolla. ¿Me entiendes? Ahora estamos ya, tenemos, no solo la cultura Colombiana, también tenemos la europea y tenemos también de Suramérica, Centroamérica, se ha vuelto colonias de varios lugares (SE La voz de las islas 1).

Además, ellos se refieren a celebraciones nacionales que no eran tomadas como teniendo un significado fundamental o haberse convertido en una imposición cultural y, no obstante los intereses de los separatistas de prohibir dichas expresiones, ellos se asocian más con la apropiación de dichas formas culturales a manera de que sean negociadas:

Raizal 2: A nosotros en los colegios nunca nos enseñaron que vamos a celebrar el día de independencia de Colombia. 'Vamos a celebrar el 20 de Julio', pero nunca nos habían dicho que es el día de la independencia que vamos a celebrar, sino que eso se volvió una fiesta ya nuestra. Eso se volvió algo cultural, algo como nuestro, nosotros hemos adoptado como nuestro, pero hay otro movimiento, hay otro movimiento que es el grupo MSD [Separatistas] que son muy radicales, entonces que todas esas costumbres que nosotros tenemos de ancestrales, que hemos venido haciendo ancestralmente, ellos quieren de una noche a la mañana borrarlo, borrarlo y que nosotros no participemos en eso (SE La voz de las islas 1).

Esto también sirve como evidencia de su ubicación como raizales, separados del concepto de colombianidad. Tal como se vio en la entrevista con el doble, y a través de este comentario, ellos se distancian a sí mismos como raizales, vinculándose de forma más cercana con otros grupos afro-caribeños, y tomando distancia de la perspectiva continental colombiana, la cual ven como una imposición cultural y lingüística. Su énfasis, tanto en su entrevista con el doble como en la semi-estructurada, sobre los vínculos con la costa este de Centroamérica y otras islas caribes es bastante diciente. Al referirse a un programa de televisión en el que trabajaron juntos, afirman que:

Raizal 2: Este programa fue un éxito total, un éxito rotundo. Había muchas expectativas y creo que se cumplieron: las expectativas de ese programa de mostrar al pueblo San Andresano que las costumbres que tenemos nosotros, paralelamente hay esas costumbres en otras regiones del Caribe, donde habitan los descendientes Afroamericanos, podríamos decirlo, afro-americanos por ser el continente, y sobre todo en Jamaica que tenemos una identidad muy estrecha (LI La voz de las islas 1, p. 5).

Raizal 1: Bueno, la mayoría de las islas en el Caribe y países que tienen ciudades en el caribe ¿no?, hablamos casi el mismo inglés criollo. Hablamos el mismo inglés criollo, tenemos casi la misma cultura, eh, sobretodo la parte gastronómica (Ll La voz de las Islas 1, p. 5).

Fuera de esto, otro tipo de contenido que aparece en su programa radial es la música, que también toma su idioma como elemento principal. Ellos se identifican con los ritmos afro-caribeños, en especial el Soka, Calypso, y en particular el Reggae, Sin embargo, incluso en la sección de música ellos hacen concesiones lingüísticas: 
Raizal 1: Igual como yo también tengo un programa los fines de semana que se llama Feelin' Eiry que es de música, y la música el 99\% de la música siempre es entre creole e inglés, pero siempre como yo sé que tenemos audiencia de habla hispana también le ponemos reggae en español que tiene mensajes muy positivos, hacia el amor, hacia la unión, como debemos de tratarnos siempre en una hermandad (Ll La voz de las islas 1, p. 3).

Otro aspecto relevante es que ellos conciben su programa como representativo de la voz de la comunidad en aspectos sociales y políticos que los afectan. Sus programas ven la ciudadanía como algo activo, y definen su trabajo como denuncia ciudadana y gestores de conciencia social, así como proveedores de espacios de discusión pública. Ellos hacen esto evidente en su descripción de sus labores en producción tanto en la entrevista con el doble como en la semi-estructurada:

Raizal 2: Lo importante es que no somos dos loros ahí únicamente hablando, sino que participa la comunidad, y la comunidad raizal, que no maneja, sobretodo, aquellos que no manejan bien el español, sobretodo en la audiencia del programa 'Al a wii da wan' que son personas de cuarenta años hacia arriba, que son las amas de casa que están en sus hogares preparando sus alimentos, son aquellas que ya están pensionadas que ya están en su casa (SE La voz de las islas 1).

Raizal 2: ['Al a wii da Wan' is] un programa que va dirigido especialmente a la comunidad nativa, pero igual hay muchos continentales que entienden y hablan nuestro creole y también nos apoyan con los diferentes temas que tenemos en la mañana en el programa, y este programa va dirigido a todo público y los temas en sí, son basados de acuerdo a lo que va pasando y transcurriendo en el día (...). Otra cosa, tampoco estamos con el gobierno y tampoco somos opositores, ¿̇entiendes? Porque la idea es trabajar en conjunto, o sea y también vistamos los diarios de nuestra isla, y visitamos tanto la oposición como el gobierno, a la gente particular, a la gente privada, todos los que viven dentro de la región, todos. Porque cuando le ocurre algo a uno, le ocurre a todos (SE La voz de las islas 1).

\section{Conclusiones}

Es evidente que la radio se convierte en una herramienta que permite fortalecer los rasgos culturales y la validez de lo local de las comunidades analizadas. Así sea una estructura mediática externa a ellos, es interesante ver cómo se apropian de ella al pensarla y construirla bajos sus necesidades y, de este modo, generar una producción bajo los aspectos que ellos consideran importantes y más valiosos para sus contextos y realidades. Tal como lo explica Rodríguez (2006), los medios comunitarios se convierten en "un espacio comunicativo que te da la libertad para jugar con lo simbólico, para re-inventarte, para recodificar tu entorno, tu cultura y tu futuro. De esta forma, identidades alternativas, imaginarios colectivos alternativos y formas alternativas de socializar encuentran una esfera pública donde surgir" (p. 5).

Asimismo, lo que han permitido este tipo de iniciativas comunicativas en algunas regiones de Colombia es promover y exponer culturas que no han sido muy reconocidas en los medios hegemónicos del país, buscando desconfigurar el orden instituido y do- 
minante en distintos niveles, y repensar una nueva manera de reconstruir lo público y lo ciudadano, como se resalta sobre todo en el caso de la comunidad Nasa en el Cauca donde se nota claramente una postura política y cultural muy clara por rescatar y hacer valer sus derechos como comunidades indígenas (de igual manera que el caso Inuit planteado en Evans, 2002). Estos grupos minoritarios han confirmado que el horizonte de deseo, explicado por Gutiérrez (2008, p. 47) como "una metáfora de lo que es colectivamente deseable y posible de construir, que habilite un sentido común de las múltiples acciones colectivas", se puede llevar a cabo desde medios de comunicación propios que se trabajen en su lengua, sus lógicas y su cosmovisión y rompan con los discursos y códigos impuestos por los medios masivos de comunicación que muchas veces se encargan de promover un discurso de patrones culturales generales para todo el país.

La radio les ha permitido a estos grupos ejercer de manera mucho más directa un control ciudadano sobre los procesos públicos que afectan directamente su región, motivando al ciudadano a tener un papel más activo y participativo dentro de su comunidad. Esto, a su vez, permite fortalecer lazos culturales y tejido social en las comunidades, puesto que abre un espacio local y propio de construcción y reconocimiento de la región. Las tres comunidades utilizan la herramienta radial para intercambiar conocimientos, validar sus saberes ancestrales, resaltar sus valores culturales y evidenciar sus necesidades locales.

Al analizar las negociaciones de identidad que se producen en cada uno de los medios estudiados se puede percibir que las tres comunidades se inclinan más por llevar a cabo identidades de tipo negociado y asumido.

La comunidad Wayuu con la radio Jujunula Makuira se alinea más hacia una identidad asumida, debido a que aunque tiene alguna producción en su lengua casi toda la programación se concentra en el idioma español y a la vez sus formatos y contenidos radiales evidencian la influencia de una cultura externa a la propia.

La comunidad Nasa con Radio Nasa y la comunidad Raizal con su espacio radial en La Voz de la Islas son ejemplo de identidades negociadas debido a que su programación y producciones radiales muestran cómo ellos se han pensado y reconocido como pueblos diferentes pero que no van en oposición a la cultura mayoritaria, sino que, por el contrario, construyen procesos que les permiten evidenciar sus rasgos culturales propios involucrando, y no excluyendo, a poblaciones distintas a las suyas. Para los productores de Radio Nasa es importante que sus contenidos e información no solo estén al alcance de la población indígena sino que también puedan ser escuchados y comprendidos por la población campesina. Esto puede, quizá, perjudicar la manutención de su lengua, pero promueve su reconocimiento y la posibilidad de fortalecerse como pueblo en la región. Igualmente, la comunidad Raizal evidencia la importancia de defender su lengua y sus rasgos culturales pero sin dejar por fuera la posibilidad de que otras culturas también hagan parte de su contenido y debate, lo que muestra que es una construcción distinta pero no excluyente.

Estas dos negociaciones, ambas incluyentes, evidencian posturas opuestas frente a la presencia de la lengua. Aunque ambas presentan formas de adaptar la estructura mediática 
radial, sus negociaciones toman posturas diferentes frente a la necesidad de dar espacio a sus perspectivas de mundo. El sacrificio lingüístico Nasa se ve como una necesidad para su cobertura como esfera pública, mientras que los raizales ven ese espacio radial como un elemento en el que deben expandir el dominio, en el término lingüístico, de su idioma.

No obstante, aunque las tres comunidades con las que trabajamos entienden que la radio es una herramienta útil para incentivar procesos participativos y culturales es evidente que "aquello que justifica la creación de los medios de comunicación en idiomas minoritarios no es necesariamente lo mismo que aquello que ayude a mantenerlos" (Cormack, 2005, p. 113) puesto que ninguna de las tres comunidades cuenta con un plan lingüístico en la emisora, ni con una guía de aspectos clave a tener en cuenta en la producción radial diaria para lograr proteger el idioma minoritario.

Cabe resaltar que en Colombia es necesario seguir investigando sobre el campo de la comunicación indígena y étnica para poder profundizar y comprender de mejor manera los rasgos de esta comunicación y la utilidad de los medios locales en estas comunidades. Debemos continuar investigando en este tema, entendiendo que "los medios locales pueden ayudar a transformar a los pueblos indígenas de consumidores de medios en productores de su propio contenido mediático" (Rodríguez y El'Gazi, 2007, p. 247) lo que lleva a que se conviertan en herramientas muy útiles para reafirmar tradiciones, renovar valores culturales, validar formatos y contenidos locales y promover la participación equitativa de las comunidades, aportándole al desarrollo local y humano del país.

\section{Referencias}

Browne, D. R. (2005). Ethnic Minorities, Electronic Media and the Public Sphere: a Comparative Approach. Euricom monographs (pp. viii, 255p.). Cresskill, N.J.: Hampton Press.

Browne, D. R., E Uribe-Jongbloed, E. (2013). Ethnic/Linguistic Minority Media: What heir History reveals, How Scholars have studied Them, and What We might ask next. In E. H. G. Jones \& E. Uribe-Jongbloed (Eds.), Social Media and Minority Languages: Convergence and the Creative Industries (pp. 1-28). Bristol, Buffalo y Toronto: Multilingual Matters.

Castells, M. (2005). The Network Society : a Cross-Cultural Perspective (pp. xx, 464p.). Cheltenham: Edward Elgar.

Castells-Talens, A., Ramos Rodríguez, J. M., E Chan Concha, M. (2009). Radio, control, and indigenous peoples: The failure of state-invented citizens' media in Mexico. Development in Practice, 19(4-5), 525-537. doi:10.1080/09614520902866298

Cormack, M. (1998). Minority Language Media in Western Europe. European Journal of Communication, 13(1), 33-52.

Cormack, M. (2004). Developing Minority Language Media Studies. Mercator Media Forum, 7(1), 3-12.

Cormack, M. (2005). The cultural politics of minority language media. International Journal of Media and Cultural Politics, 1 (1), 107-122

Cormack, M. (2007). The Media and Language Maintenance. In M. Cormack \& N. Hourigan (Eds.), Minority Language Media (pp. 52-68). Clevedon: Multilingual Matters. 
Cormack, M. (2012). Towards an Understanding of Media Impact on Minority Language use. In E. H. G. Jones \& E. Uribe-Jongbloed (Eds.), Social Media and Minority Languages: Convergence and the Creative Industries (pp. 255-265). Bristol, Buffalo and Toronto: Multilingual Matters.

Cuesta Moreno, O. (2012a). Investigaciones radiofónicas : de la radio a la radio indígena . Una revisión en Colombia y Latinoamérica. Anagramas, 10(20), 181-196.

Cuesta Moreno, O. (2012b). Investigaciones radiofónicas, Ánfora, 19(33), 165-183.

Cunningham, S. (2001). Popular Media as Public "Sphericules" for Diasporic Communities. International Journal of Cultural Studies, 4(2), 131-147.

Evans, M. R. (2002). Hegemony and Discourse: Negotiating Cultural Relationships through Media Production. Journalism, 3(3), 309-329.

Forde, S., Foxwell, K., \& Meadows, M. (2009). Developing dialogues: Indigenous and ethnic community broadcasting in Australia. Bristol; Chicago: Intellect.

Garcia-Canclini, N. (2004). Diferentes, desiguales y desconectados: Mapas de la interculturalidad. Buenos Aires: Gedisa, Editorial, S. A.

Gitlin, T. (1998). Public Sphere or Public Sphericules? In T. Liebes \& J. Curran (Eds.), Media, Ritual and Identity (pp. 168-174). London \& New York: Routledge.

Gutiérrez, R. (2008). Los ritmos del Pachakuti: Movilización y levantamiento indígena popular en Bolivia. Buenos Aires: Tinta Limón.

Husband, C. (1994). A richer vision: The development of ethnic minority media in western democracies. Communication and development series (p. x, 149 p.). Paris, France E London, UK: Unesco Pub. E J. Libbey.

Husband, C. (2005). Minority Ethnic Media as Communities of Practice: Professionalism and Identity Politics in Interaction. Ethnic and Migration Studies, 31(3), 461-479. doi: 10.1080/13691830500058802

Lewis, M. P., Simons, G. F., E Fenning, C. D. (Eds.). (2013). Ethnologue: Languages of the World (Seventeent.). Dallas, Texas: SIL International. Retrieved from http://ethnologue.org

Meadows, M. H. (1992). A watering can in the desert: Issues in indigenous broadcasting policy in Australia. Brisbane: Institute for Cultural Policy Studies, Griffiths University.

Meadows, M. H. (2009). Walking the Talk: Reflections of Indigenous Audience Research Methods. Participations: Journal of Audience E Reception Studies, 6(1), 118-136.

Meadows, M. H., E Molnar, H. (2002). Bridging the Gaps: Towards a History of Indigenous Media in Australia. Media History, 8(1), 9-20.

Murillo, M. (2008). Weaving a Communication Quilt in Colombia: Civil Conflict, Indigenous Resistance and Community Radio in Northern Cauca. In P. Wilson \& M. Stewart (Eds.), Global Indigenous Media: Cultures, Poetics, and Politics (pp. 145-159). Durham E London: Duke University Press.

Nicolini, D. (2009). Articulating Practice through the Interview to the Double. Management Learning, 40(2), 195-212. doi:10.1177/1350507608101230

ONIC. (2009). Diagnóstico Integral de Emisoras y/o Radios Indígenas. Bogotá: ONIC.

Pavlenko, A., E Blackledge, A. (2003). Introduction: New Theoretical Approaches to the Study of the Negotiation of Identities in Multilingual Contexts. In A. Pavlenko \& A. Blackledge (Eds.), Negotiations of Identity in Multilingual Contexts (pp. 1-33). Clevedon, Buffalo, Toronto \& Sidney: Multilingual Matters. 
Riggins, S. H. (1992). The Promise and Limits of Ethnic Minority Media. In S. H. Riggins (Ed.), Ethnic Minority Media (pp. 276-288). Newbury Park et al.: SAGE.

Rodriguez, C., E El'Gazi, J. (2007). La Poética de la Radio Indígena en Colombia. En O. Rincón, M. P. Saffón, A. Cadavid, A. E. Villegas, N. Martínez, M.I. Andrade... (Autores) Ya no es posible el silencio (pp. 239-262). Bogotá: Centro de Competencia en Comunicación en América Latina - Friederich Ebert Stiftung

Rodríguez, C. (2006). Tres Lecciones Aprendidas de los Medios Ciudadanos y Comunitarios en Colombia. Ponencia presentada en el Encuentro Nacional de Medios Ciudadanos. Bogotá: Ministerio de Cultura

Unidad de Radio. (2002). Memorias del Encuntro Internacional de Radios Indígenas de América. Bogotá: Ministerio de Cultura.

Wenger, E. (1998). Communities of Practice: Learning, Meaning, and Identity. Learning in doing (p. xv,318p). Cambridge: Cambridge University Press.

Wilson, P., E Stewart, M. (2008). Indigeneity and Indigenous Media on the Global Stage. In P. Wilson \& M. Stewart (Eds.), Global Indigenous Media (pp. 1-35). Durham \& London: Duke University Press. 